\title{
Ouverture et équilibre dans les procédures déontologiques
}

\section{Jean Martin}

Dr med., membre de la rédaction

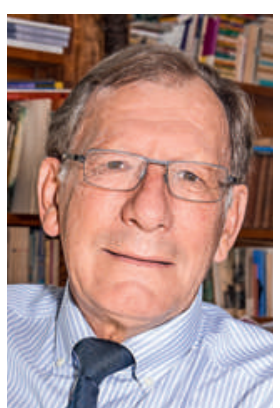

Importante contribution dans le premier numéro de 2016 du BMS [1]: les actions et orientations de la FMH décrites par S. Cueni et al. devraient apporter une amélioration significative des habitudes antérieures, qui impliquaient de sérieuses difficultés pour les patientes [2]. Qu'il soit permis d'évoquer certaines réalités: dans les années 1980, comme médecin cantonal, je me suis adressé au collègue d'un canton voisin à propos d'un médecin autorisé dans son canton qui souhaitait pratiquer sur Vaud. Nous nous sommes vus répondre que la protection des données interdisait de nous renseigner! Situation pour le moins ennuyeuse du point de vue de notre mandat de protection de la santé du public: à supposer que ce confrère ait retenu défavorablement l'attention pour une raison quelconque auparavant, devions-nous attendre, cas échéant, qu'il se fasse remarquer défavorablement chez nous? Autre situation délicate: nous avons appris par la presse qu'un professionnel de santé était condamné pour complicité de crime dans un autre canton mais celui-ci contestait la sanction prise à l'endroit de son autorisation de pratiquer sur Vaud. Dans de tels cas, on voit combien, dans une société de plus en plus mobile, il est nécessaire d'assurer une communication adéquate entre instances concernées. A l'époque, devant des obstacles du genre "juridisme étroit», restait la possibilité de contacts personnels informels et, si nous voulions assumer notre responsabilité, nous devions y recourir. Cela pouvait permettre de sauvegarder l'essentiel mais dépendait des rapports de confiance avec nos homologues.

Des questions comparables se posent au sein du canton; ainsi entre la commission de déontologie de la société médicale et le médecin cantonal. Entre gens de bonne compagnie, on comprenait le besoin de faire part à l'autorité de surveillance d'éléments graves concernant un professionnel mais cela n'était pas réglé formellement. Il est pratique, je l'ai souvent dit, que (co-) existent des procédures disciplinaires privées et publiques. Le privé s'occupe de manière plus expéditive de litiges concernant les membres de la corporation entre eux, dont l'expérience montre au reste qu'ils peuvent être de gravité objective modeste. Par contre, quand des manquements sérieux, des cas de mal- pratique ou de gestes très inadéquats (entre autres à connotation sexuelle) parviennent à la commission associative, des modalités de communication doivent être prévues avec l'autorité cantonale. A cet égard, il convient de saluer les démarches actuelles au sein de la FMH, en espérant qu'on ne s'arrêtera pas en chemin dans leur mise en œuvre - comme Cueni et al. le notent, jusqu'ici les commissions de déontologie font «trop rarement usage des sanctions à leur disposition». Ces auteurs rappellent à très juste titre cet élément du droit administratif, qui était appliqué par analogie en pratique déontologique associative, selon quoi la plaignante n'est pas considérée comme partie et, en conséquence, n'avait pas accès au contenu de l'instruction. De plus, au terme de cette dernière, elle était simplement informée qu'une décision avait été prise, sans en connaitre la teneur. Situation anachronique et surtout déséquilibrée (pour acquérir le statut de partie, il fallait alors aller vers une procédure pénale, avec ses coûts et délais). Merci à la Chambre médicale de la FMH d'avoir corrigé cette situation par sa décision de 2013.

Nous nous sommes vus répondre que la protection des données interdisait de nous renseigner!

Les orientations décrites par Cueni et al. sont nécessaires et opportunes. Y compris s'agissant du principe d'échanges entre commissions de sociétés cantonales, de journées de travail et d'une banque de données nationale.

Un point encore: la question se posait à l'époque de savoir si un professionnel interpelé pouvait «s'abriter» derrière le secret médical. Ce qui a amené l'inclusion dans la loi vaudoise sur la santé publique d'une disposition (art. 80a) fixant que cette personne «ne peut pas se prévaloir du secret professionnel pour refuser de renseigner les autorités sanitaires sur les faits dont elle est elle-même accusée, ni pour refuser de témoigner au plan civil dans des conflits l'opposant à ses patients».

\section{Références}

1 Cueni S, Hauri R, Romann C. Activité médicale et dignité humaine - La FMH opte pour une nouvelle voie. Bull Méd Suisses. 2016;97(1):9-10.

2 Comme Cueni et al., nous optons ici pour l'usage du féminin les femmes étant plus souvent concernées. 